\title{
ORTHOGONALITY RELATION FOR FROBENIUS- AND QUASI-FROBENIUS-ALGEBRAS
}

\section{TADASI NAKAYAMA}

The celebrated orthogonality relation for the coefficients of the regular representation of a group was extended first to the modular case by Nesbitt, and then to Frobenius-algebras by the writer; the proof was reproduced in [4] $]^{1}$ together with a second proof. Another proof of this orthogonality, for the coefficients of the regular representations of Frobenius-algebras, and its interesting application to faithful representations were given by Brauer [1]. In the present note we propose a still different proof, and generalize the orthogonality to quasi-Frobenius-algebras.

1. A class of automorphisms in a Frobenius-algebra. Let $\mathfrak{A}$ be a Frobenius-algebra $[1 ; 2 ; 3 ; 4$, over a field $\Omega$, and let

$$
\left(a_{1}, a_{2}, \cdots, a_{n}\right) \quad \text { with } a_{\sigma} a_{\tau}=\sum_{\iota} \alpha_{\sigma \tau \iota} a_{\iota}
$$

be its basis. Let

$$
P=\left(\sum_{\imath} \alpha_{\sigma \tau \iota} \mu_{\imath}\right)
$$

be a nonsingular parastrophic matrix. Let

$$
L(x)=\left(\lambda_{\sigma \tau}(x)\right), \quad R(x)=\left(\rho_{\sigma \tau}(x)\right)
$$

be the left and right regular representations defined by the basis (1). We have

$$
R(x) P=P L(x) .
$$

With $x=\sum \xi_{\iota} a_{\imath} \in \mathcal{Y}$ we put $[2, \mathrm{II}, \S 1]$

$$
x^{*}=\sum \xi_{\imath}^{*} a_{\imath}, \quad\left(\xi_{\imath}^{*}\right)=\left(P^{\prime}\right)^{-1} P\left(\xi_{\iota}\right) .
$$

In particular

$$
\left(a_{1}^{*}, a_{2}^{*}, \cdots, a_{n}^{*}\right) P^{-1}=\left(a_{1}, a_{2}, \cdots, a_{n}\right)\left(P^{\prime}\right)^{-1} .
$$

* is an automorphism of $\mathfrak{A}$, and we have further, as a counterpart to (4),

$$
R(x) P^{\prime}=P^{\prime} L\left(x^{*}\right) .
$$

Received by the editors April 9, 1951.

${ }^{1}$ Numbers in brackets refer to the references at the end of the paper. 
Also

$$
\begin{aligned}
\left(x^{*}\left(a_{1}^{*}, a_{2}^{*}, \cdots, a_{n}^{*}\right) P^{-1}\right. & =) x^{*}\left(a_{1}, a_{2}, \cdots, a_{n}\right)\left(P^{\prime}\right)^{-1} \\
& =\left(a_{1}, a_{2}, \cdots, a_{n}\right) L\left(x^{*}\right)\left(P^{\prime}\right)^{-1} \\
& =\left(a_{1}, a_{2}, \cdots, a_{n}\right)\left(P^{\prime}\right)^{-1} R(x) \\
( & \left.=\left(a_{1}^{*}, a_{2}^{*}, \cdots, a_{n}^{*}\right) P^{-1} R(x)\right) \\
\left(\left(P^{\prime}\right)^{-1}\left(a_{\imath}^{*}\right) x\right. & =) P^{-1}\left(a_{\imath}\right) x=P^{-1} R(x)\left(a_{\imath}\right) \\
& =L(x) P^{-1}\left(a_{\imath}\right)\left(=L(x)\left(P^{\prime}\right)^{-1}\left(a_{\imath}^{*}\right)\right) .
\end{aligned}
$$

Thus, if we put

$$
\left(b_{1}, b_{2}, \cdots, b_{n}\right)=\left(a_{1}, a_{2}, \cdots, a_{n}\right)\left(P^{\prime}\right)^{-1}=\left(a_{1}^{*}, a_{2}^{*}, \cdots, a_{n}^{*}\right) P^{-1}
$$

then

$$
\begin{aligned}
x^{*}\left(b_{1}, b_{2}, \cdots, b_{n}\right) & =\left(b_{1}, b_{2}, \cdots, b_{n}\right) R(x), \\
\left(b_{\iota}\right) x & =L(x)\left(b_{\iota}\right) .
\end{aligned}
$$

We call $\left(b_{1}, b_{2}, \cdots, b_{n}\right)$ conjugate to $\left(a_{1}, a_{2}, \cdots, a_{n}\right)$.

With an arbitrary representation $Z(x)$ of $\mathfrak{A}$, consider the matrix

$$
Z=\sum Z\left(b_{\imath}\right) a_{\imath}
$$

in $\mathscr{A}$. We have

$$
\begin{aligned}
3 x & =\sum Z\left(b_{\iota}\right) a_{\iota} x=\sum_{\iota} Z\left(b_{\iota}\right) \sum_{\tau} \rho_{\iota \tau}(x) a_{\tau} \\
& =\sum_{\tau} Z\left(\sum_{\iota} b_{\iota} \rho_{\iota \tau}(x)\right) a_{\tau}=\sum Z\left(x^{*} b_{\tau}\right) a_{\tau} \\
& =\sum Z\left(x^{*}\right) Z\left(b_{\tau}\right) a_{\tau}=Z\left(x^{*}\right) 3 .
\end{aligned}
$$

Similarly we have, by virtue of (12) instead of (11),

$$
x 8=3 Z(x) \text {. }
$$

A different choice of nonsingular parastrophic matrix gives rise to an automorphism congruent to ${ }^{*}$ modulo inner automorphisms. On the other hand, if we start with another basis $\left(a_{1}, a_{2}, \cdots, a_{n}\right) Q$ of I, then the parastrophic matrix $Q^{\prime} P Q$ belonging to it gives the same automorphism *. Take the second basis such that the left regular representation defined by it assumes a reduced form 


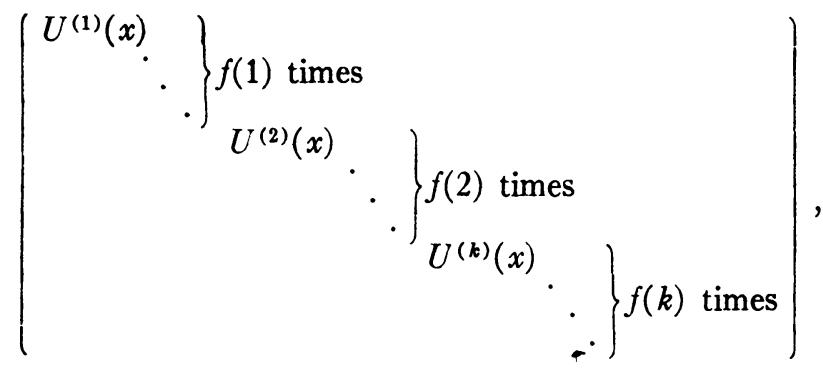

$$
\begin{aligned}
& U^{(\kappa)}(x)=\left(\begin{array}{lll}
G^{(\kappa)}(x) & & M^{(\kappa)}(x) \\
& \ddots & \\
& & F^{(\kappa)}(x)
\end{array}\right)
\end{aligned}
$$

with directly indecomposable $U^{(\kappa)}$ and irreducible $G^{(\kappa)}, F^{(\kappa)}$. Here $G^{(\kappa)}(x)$ is equivalent to $F^{(\kappa)}\left(x^{\left(*^{-1}\right)}\right)$; cf. [2, II, \$1]. For those $\kappa$ for which $U^{(\kappa)}$ are not irreducible, we can assume that our basis is chosen so that $G^{(\kappa)}(x)=F^{(\kappa)}\left(x^{\left(*^{-1}\right)}\right)$. As for those $\kappa$ for which the $U^{(\kappa)}$ are irreducible, we see easily that we can choose $\mu_{\tau}$ in (2) suitably so that $F^{(\kappa)}(x)=F^{(\kappa)}\left(x^{\left(*^{-1}\right)}\right)$; those $\kappa$ correspond to simple subalgebras of $\mathfrak{A}$ which are direct components of $\mathfrak{A}$, and these components together form a semisimple (hence certainly symmetric) algebra, and we have merely to choose our ${ }^{*}$ such that it induces the identity automorphism on this subalgebra. Under these adjustments we have

$$
U^{(\kappa)}(x)=\left(\begin{array}{lll}
F^{(\kappa)}\left(x^{\left(*^{-1}\right)}\right) & & M^{(\kappa)}(x) \\
& \cdot & \\
& \cdot F^{(\kappa)}(x)
\end{array}\right) .
$$

\section{Orthogonality relation for Frobenius-algebras.}

THEOREM 1. If a representation $Z(x)$ of $\mathfrak{A}$ does not contain $U^{(\kappa)}(x)$, then $F^{(\kappa)}(z)=0$ for every element $z$ of the matrix $\mathbb{3}$ in (13). (If a representation contains $U^{(\kappa)}$, then it contains $U^{(\kappa)}$ as a direct component; cf. [3, \$2, Remark 3].)

For, let $\mathfrak{l}$ be the linear space spanned, not necessarily linearly independently, by the elements of the row of 3 containing our $z$. It is a left-ideal of $\mathfrak{A}$, as (15) shows. Suppose, contrary to our assertion, $F^{(x)}(z) \neq 0$. Then $\mathfrak{l}$ contains a certain primitive idempotent element $e$ which generates a left-ideal $\mathscr{N} e$ defining $U^{(\kappa)}$. But $\mathfrak{l}$ is a homomorphic image of the representation module $z$ of $Z(x)$, again by (15). Hence $\mathfrak{A} e$ is a submodule of a homomorphic image of $z$, which means that $Z$ contains $U^{(\kappa)}$, contrary to our assumption. 
In particular,

THEOREM $1^{\prime}$. If $\zeta(x)$ is a coefficient in the representation $U^{(\lambda)}$ and $\lambda \neq \kappa$, then $F^{(\kappa)}\left(\sum \zeta\left(b_{\imath}\right) a_{\imath}\right)=\sum \zeta\left(b_{\imath}\right) F^{(\kappa)}\left(a_{\imath}\right)=0$.

Further, the part of $U^{(\kappa)}$ complementary to $F^{(\kappa)}$ forms a representation which certainly does not contain $U^{(k)}$. The same is the case with the part complementary to $G^{(\kappa)}$. Thus we have the following theorem.

THEOREM $1^{\prime \prime}$. If $\zeta(x)$ is a coefficient in the representation $U^{(x)}$ which is not in $M^{(\kappa)}$, then $F^{(\kappa)}\left(\sum \zeta\left(b_{\imath}\right) a_{\imath}\right)=\sum \zeta\left(b_{\imath}\right) F^{(\kappa)}\left(a_{\imath}\right)=0$.

REMARK. In Theorem $1^{\prime \prime}$ only the reduced form (16) of $U^{(\kappa)}$ is used, and the more specified form (17) of $U^{(x)}$ is unnecessary. In Theorem $1^{\prime}, U^{(\kappa)}$ can of course be in any form, not necessarily reduced.

We now assume that the $F^{(\kappa)}$ are absolutely irreducible, or at least that a certain $F^{(\kappa)}$, which we deal with, is so. Denote the degree of $U^{(\kappa)}$ by $u(\kappa)$. Because of our assumption the degree of $F^{(\kappa)}$ is equal to the multiplicity $f(\kappa)$ of $U^{(\kappa)}$ in the left regular representation; cf. (16). Construct

$$
\mathfrak{U}^{(\kappa)}=\sum U^{(\kappa)}\left(b_{\imath}\right) a_{\imath}
$$

It satisfies

$$
\mathfrak{U}^{(\kappa)} x=U^{(\kappa)}\left(x^{*}\right) \mathfrak{U}^{(\kappa)}, \quad x \mathfrak{U}^{(\kappa)}=\mathfrak{U}^{(\kappa)} U^{(\kappa)}(x) .
$$

It is well known, and easy to see, that $u(\kappa)$ coefficients of the first row (or any one of the first $f(\kappa)$ rows) of $U^{(\kappa)}$ are linearly independent. (As a matter of fact, it is also well known that the coefficients in the first $f(\kappa)$ rows of $U^{(\kappa)}$ altogether are linearly independent. But this may be seen from the sequel and need not be assumed as known.) Hence the $u(\kappa)$ elements in the first row of $U^{(\kappa)}$ are linearly independent. They form, according to (19), a left-ideal $\mathfrak{l}_{1}^{(\kappa)}$ which defines $U^{(\kappa)}$. $\mathfrak{l}_{1}^{(\kappa)}$ is a direct component of $\mathfrak{A}$. Moreover, the first $u(\kappa)-f(\kappa)$ of our elements of the first row of $U^{(\kappa)}$ form a (unique) maximal left-subideal of $\mathfrak{I}_{1}^{(\kappa)}$, as the reduced form of $U^{(\kappa)}$ shows; this follows also readily from Theorems $1^{\prime}, 1^{\prime \prime}$. Here the maximal left-subideal is precisely the intersection of $\mathfrak{l}_{1}^{(\kappa)}$ with the radical $\mathfrak{N}$ of $\mathfrak{A}$. Similarly the second, the third, $\cdots$, the $f(\kappa)$ th rows of $U^{(\kappa)}$ span left-ideals $\mathfrak{l}_{2}^{(\kappa)}, \mathfrak{l}_{3}^{(\kappa)}, \cdots$, $\mathfrak{l}_{f(\kappa)}^{(\kappa)}$ belonging to $U^{(\kappa)}$.

Analogously, the last $f(\kappa)$ columns (i.e., the $u(\kappa)-f(\kappa)+1$ st, $u(\kappa)$ $-f(\kappa)+2$ nd, $\cdots, u(\kappa)$ th columns) of $\mathfrak{u}^{(\kappa)}$ span right-ideals $\mathfrak{r}_{1}^{(\kappa)}, \mathfrak{r}_{2}^{(\kappa)}, \cdots, \mathfrak{r}_{f(\kappa)}^{(\kappa)}$; all belonging to the representation $U^{(\kappa)}\left(x^{*}\right)$.

Now, take a system $\left\{e_{\xi j}^{(\kappa)}\right\}$ of elements in $\mathfrak{A}$ such that 


$$
F^{(\kappa)}\left(e_{i j}^{(\kappa)}\right)=\left(\begin{array}{cc}
0 & 0 \\
& 1 \\
0 & 0
\end{array}\right)(1 \text { at }(i, j)), \quad F^{(\lambda)}\left(e_{i j}^{(\kappa)}\right)=0 \quad(\lambda \neq \kappa) .
$$

The elements $e_{i j}^{(\alpha)}$ form a system of matric units modulo $\mathfrak{N}$. We have $\mathfrak{U}^{(\kappa)} e_{\mathfrak{y}}^{(\kappa)}=U^{(\kappa)}\left(e_{\mathfrak{j}}^{(\kappa) *}\right) \mathfrak{U}^{(\kappa)}$. By virtue of (17), which we make use of for the first time, this gives

$$
\mathfrak{l}_{1}^{(\kappa)} e_{1 i}^{(\kappa)} \equiv \mathfrak{l}_{i}^{(\kappa)} \bmod \mathfrak{N}
$$

more precisely

$$
\left(1 \text { st row of } \mathfrak{U}^{(\kappa)}\right) e_{1 i}^{(\kappa)} \equiv\left(i \text { th row of } \mathfrak{U}^{(\kappa)}\right) \bmod \mathfrak{R} \text {. }
$$

Similarly we have

$$
e_{i 1}^{(\kappa)} \stackrel{(k)}{\mathfrak{r}} \equiv \mathfrak{r}_{1}^{(k)} \bmod \mathfrak{N}
$$

and in fact

$$
\begin{aligned}
& e_{i 1}^{(\kappa)}\left(u(\kappa)-f(\kappa)+1 \text { st column of } \mathfrak{U}^{(\kappa)}\right) \\
& \equiv\left(u(\kappa)-f(\kappa)+i \text { th column of } \mathfrak{U}^{(\kappa)}\right) \bmod \mathfrak{N}
\end{aligned}
$$

Thus the $(1, u(\kappa)-f(\kappa)+1)$-element of $\mathfrak{U}^{(\kappa)}$ lies in $e_{11}^{(\kappa)} \mathfrak{A} e_{11}^{(\kappa)} \bmod \mathfrak{N}$, and is, therefore, congruent to $\xi^{(\kappa)} e_{11}^{(\kappa)} \bmod \mathfrak{N}$ with $\xi^{(\kappa)} \in \Omega$. The $(i, u(\kappa)-f(\kappa)+j)$-element of $\mathfrak{u}^{(\kappa)}$ is then congruent to $\xi^{(\kappa)} e_{i j}^{(\kappa)} \bmod \mathfrak{R}$, according to (22), (24). Its representation matrix in $F^{(\kappa)}$ is the matrix with $\xi^{(\kappa)}$ at $(i, j)$ and 0 's elsewhere. This means, if we put

$$
F^{(\kappa)}(x)=\left(\phi_{s t}^{(\kappa)}(x)\right)_{s t}, \quad M^{(\kappa)}(x)=\left(\mu_{i j}^{(\kappa)}(x)\right)_{i j},
$$

that

$$
\begin{aligned}
\phi_{s t}^{(\kappa)}\left(\sum_{\iota} \mu_{i j}^{(\kappa)}\left(b_{\imath}\right) a_{\imath}\right)=\sum_{\iota} \phi_{s t}^{(\kappa)}\left(a_{\imath}\right) \mu_{i j}^{(\kappa)}\left(b_{\imath}\right)=\xi^{(\kappa)} \delta_{s j} \delta_{t i} \\
\\
\quad\left(\xi^{(\kappa)}(\in \Omega) \neq 0\right) .
\end{aligned}
$$

Of course the relations in Theorems $1,1^{\prime}, 1^{\prime \prime}$ can be written as

$$
\sum_{\imath} \phi_{s t}^{(k)}\left(a_{\imath}\right) \zeta\left(b_{\imath}\right)=0
$$

where $\zeta(x)$ is as in those theorems. Thus:

Theorem 2. Let $F^{(\kappa)}$ be absolutely irreducible. With (25) we have 
the orthogonality (26). If $\zeta(x)$ is as in Theorems $1,1^{\prime}, 1^{\prime \prime}$, then we have (27).

3. Quasi-Frobenius-algebras. Let now $\mathfrak{A}$ be a quasi-Frobeniusalgebra $[2 ; 4]$ with a basis

$$
\left(a_{1}, a_{2}, \cdots, a_{n}\right),
$$

which defines the left and right regular representations $L(x), R(x)$ of A. Let

$$
\left(u_{1}, u_{2}, \cdots, u_{n}\right)=\left(a_{1}, a_{2}, \cdots, a_{n}\right) Q
$$

be a second, auxiliary basis of $\mathfrak{A}$ such that the left regular representation $L_{1}(x)=Q_{1}^{-1} L(x) Q_{1}$ defined by it has a decomposed form similar to (16). Let $U_{1}^{(\kappa)}$ be its indecomposable components; $U_{1}^{(\kappa)} \simeq U^{(\kappa)}$. The right regular representation contains each $U_{1}^{(\alpha)}$ with a certain multiplicity, say $g(\kappa)$, greater than 0 (and it consists of them only). Put

$$
h(\kappa)=\min (f(\kappa), g(\kappa)) .
$$

There exists a right-ideal of $\mathfrak{A}$ which defines a representation containing (directly) each $U_{1}^{(\kappa)}$ exactly $h(\kappa)$ times. Let

$$
\left(w_{1}, w_{2}, \cdots, w_{m}\right) \quad\left(m=\sum h(\kappa) u(\kappa)\right)
$$

be its basis such that the representation defined by it has $U_{1}^{(\kappa)}$ in their order of indices, that is, first $h(1)$ times $U_{1}^{(1)}$, then $h(2)$ times $U_{1}^{(2)}$, and so on. Augment it with $(f(\kappa)-h(\kappa)) u(\kappa) 0$ 's after the $h(\kappa) u(\kappa)$ $w$ 's belonging to $U_{1}^{(\kappa)}(\kappa=1,2, \cdots, k)$. Thus we obtain a vector

$$
\left(v_{1}, v_{2}, \cdots, v_{n}\right)
$$

with $\sum(f(\kappa)-h(\kappa)) u(\kappa)$ dummies 0 , satisfying

$$
\left(v_{\imath}\right) x=L_{1}(x)\left(v_{\imath}\right) \text {. }
$$

Put

$$
\left(b_{1}, b_{2}, \cdots, b_{n}\right)=\left(v_{1}, v_{2}, \cdots, v_{n}\right) Q_{1}^{\prime} ;
$$

$\left(b_{\imath}\right)$ does not, together with $\left(v_{\imath}\right)$, form a basis of $\mathfrak{A}$, in general. We have

$$
\left(b_{\iota}\right) x=Q_{1} L_{1}(x) Q_{1}^{-1}\left(b_{\iota}\right)=L(x)\left(b_{\imath}\right) .
$$

With a representation $Z(x)$ of $\mathfrak{A}$, define $\mathbb{Z}$ as in (13) by means of this $\left(b_{\iota}\right)$ (and the basis $\left(a_{\imath}\right)$ ). Then we obtain (15) in the same way as before.

Now, consider any left regular representation of $\mathfrak{A}$ decomposed and reduced in the form (16). We can prove quite similarly that: 
TheOREM 3. With the vector $\left(b_{\mathfrak{}}\right)$ in (33), constructed as above, Theorems 1, 1', 1" are valid for the quasi-Frobenius-algebra $\mathfrak{A}$.

Consider next the matrix

$$
3^{*}=\sum Z\left(a_{\imath}\right) b_{\imath}
$$

in $\mathfrak{A}$. By means of (34) we see

$$
\mathfrak{B}^{*} x=Z(x) \mathfrak{B}^{*}
$$

This leads us to the following theorem.

TheOREM 4. Suppose that $Z$ does not contain the component $V^{(\kappa)}$ of the right regular representation having $F^{(k)}$ as its top constituent. Then $F^{(x)}(\tilde{z})=0$ for any element $\tilde{z}$ of $\mathfrak{B}^{*}$. Hence, also in our quasi-Frobeniusalgebra generalization Theorem 3 of Theorems (1 and )1', $1^{\prime \prime}$ we may interchange $\left(a_{\imath}\right)$ and $\left(b_{\imath}\right)$ provided we consider $V^{(k)}$ in place of $U^{(k)}$.

(For a Frobenius-algebra such a modification is rather meaningless.)

Now, in order to specify our basis $\left(b_{\imath}\right)$ further, we start with a (maximal) system $\left\{e_{\mathfrak{\imath}}^{(\kappa)}\right\}$ of primitive idempotent elements in $\mathfrak{A}$ such that $\mathfrak{\Re} e_{i}^{(\kappa)}$ defines $U_{1}^{(\kappa)}\left(\simeq U^{(\kappa)}\right)$. Let $\left\{c_{i j}^{(\kappa)}\right\}$ be, for each $\kappa$, a system of matric units with $c_{\mathfrak{l}}^{(\kappa)}=e_{\mathfrak{l}}^{(\kappa)}$. We assume that our auxiliary basis $\left(u_{\imath}\right)((29))$ is taken in accord with the decomposition

$$
\mathfrak{A}=\sum \mathfrak{A} e_{i}^{(\kappa)}
$$

and is composed of the bases of $e_{j}^{(\lambda)} \mathfrak{M} e_{i}^{(\kappa)}$ such that each basis of $e_{j}^{(\lambda)} \mathfrak{Q} e_{i}^{(\kappa)}$ is obtained from that of $e_{1}^{(\lambda)} \mathfrak{Q} e_{1}^{(\kappa)}$ by the left-and right-multiplications of $c_{j 1}^{(\lambda)}, c_{1 i}^{(\kappa)}$. (If the $F^{(\kappa)}$ are absolutely irreducible, this means that $\left(u_{\imath}\right)$ is a so-called Cartan basis; we may perhaps call our basis a Cartan basis in the wider sense.) Put

$$
E=\sum_{\kappa} \sum_{i=1}^{h(\kappa)} e_{i}^{(\kappa)} ;
$$

we can, and shall, take Eशr as our right-ideal possessing the basis $\left(w_{\imath}\right)((30))$. Replace in $\left(u_{\imath}\right)$ those $u^{\prime}$ s not belonging to $\mathfrak{A}_{0}=E \mathfrak{P} E$ simply by 0 's, to obtain a vector

$$
\left(y_{1}, y_{2}, \cdots, y_{n}\right) \text {; }
$$

$\left(y_{\mathfrak{}}\right)$ is essentially a basis of $\mathfrak{A}_{0}$, augmented by some 0 's. For $x_{0} \in \mathfrak{A}_{0}$ we have

$$
x_{0}\left(y_{1}, y_{2}, \cdots, y_{n}\right)=\left(y_{1}, y_{2}, \cdots, y_{n}\right) L_{1}\left(x_{0}\right) \quad\left(x_{0} \in \mathfrak{A}_{0}\right)
$$


with our left regular representation $L_{1}$ of $\mathfrak{A}$ defined by $\left(u_{\imath}\right)$. Also

$$
\left(y_{\imath}\right) x_{0}=R_{1}\left(x_{0}\right)\left(y_{\imath}\right)
$$$$
\left(x_{0} \in \mathfrak{A}_{0}\right) \text {. }
$$

$\mathfrak{A}_{0}$ is a Frobenius-subalgebra of (the quasi-Frobenius-algebra) $\mathfrak{A}$. Let $\dagger$ be its automorphism defined by a nonsingular parastrophic matrix as in $\$ 1$. Take a basis of $\mathfrak{A}_{0}$ conjugate (with respect to the same parastrophic matrix) to the basis consisting of the nonzero $y_{1}$, and augment it with 0 's at the same places where $\left(v_{\imath}\right) E$ has 0 's, to obtain

$$
\left(z_{1}, z_{2}, \cdots, z_{n}\right) \text {. }
$$

From the Cartan basis property of $\left(u_{\imath}\right)$, in the wider sense, and from the way we enlarged $\left(w_{\imath}\right)$ into $\left(v_{\imath}\right)$, we see easily (cf. also the argument below)

$$
\left(z_{\imath}\right) x_{0}=L_{1}\left(x_{0}\right)\left(z_{\imath}\right)
$$$$
\left(x_{0} \in \mathfrak{Q}_{0}\right) \text {. }
$$

It is also not difficult to see

$$
\stackrel{x}{\dagger}_{0}^{\dagger}\left(z_{1}, z_{2}, \cdots, z_{n}\right)=\left(z_{1}, z_{2}, \cdots, z_{n}\right) R_{1}\left(x_{0}\right) \quad\left(x_{0} \in \mathfrak{A}_{0}\right) .
$$

Each idempotent element $e_{j}^{(\lambda)}$ is represented in $L_{1}$ by a diagonal matrix having 1's and 0's on the diagonal, and different $e_{j}^{(\lambda)}$ have 1 's on different places (on the diagonal). The representation matrix of $c_{i j}^{(\lambda)}$ has 1's at the intersections of those rows and columns where the matrices of $e_{i}^{(\lambda)}$ and $e_{j}^{(\lambda)}$ have 1's (and 0's elsewhere). From this form of $L_{1}$ and (43) it follows that $\left(z_{\iota}\right)$ consists of the basis of the modules $\mathfrak{A}_{0} e_{j}^{(\lambda)}(1 \leqq j \leqq h(\lambda))$ (and 0 's) and the part corresponding to $\mathfrak{A}_{0} e_{j}^{(\lambda)}$ is obtained from the part corresponding to $\mathfrak{A}_{0} e_{1}^{(\lambda)}$ by the right-multiplication of $c_{1 j}^{(\lambda)}$. Replace in $\left(z_{\iota}\right)$ those 0 's, which are in the places we had basis elements of $E \mathfrak{Y}(1-E)$ in $\left(v_{\imath}\right)$, by the basis elements of $E \mathfrak{A} c_{1 i}^{(\lambda)}=\mathfrak{A}_{0} e_{1}^{(\lambda)} c_{1 i}^{(\lambda)}, i=h(\kappa)+1, \cdots, f(\lambda)$, obtained from those (in $\left(z_{\iota}\right)$ ) of $\mathfrak{A}_{0} e_{1}^{(\lambda)}$ by the (right-)multiplication of $c_{1 \mathfrak{l}}^{(\lambda)}$. Then we obtain a vector which is essentially a basis of $E \mathfrak{A}$, augmented with some 0's. Denote it $\left(v_{\imath}\right)$. It satisfies (32) (which justifies our notation). To see that we have merely to verify the relation for the elements $x$ of a form $x=c_{j 1}^{(\lambda)} x_{0} c_{11}^{(\kappa)}\left(x_{0} \in e_{1}^{(\lambda)} \mathfrak{M} e_{1}^{(\kappa)}\right)$. Thus it suffices to verify the relation for $x=c_{j 1}^{(\lambda)}, x_{0}$, and $c_{11}^{(\alpha)}$. But the relation is clear for these elements either because of the above construction of our $\left(v_{\imath}\right)$ and the structure of the representation matrices of $c_{i j}^{(\kappa)}$, mentioned above, or because of (43). So, we can employ this $\left(v_{\imath}\right)$ as our vector in (31). It satisfies, besides (32),

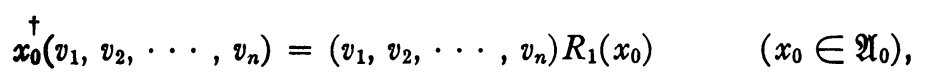


as follows from (44) and our construction of $\left(v_{\imath}\right)$.

Define $\left(b_{1}, b_{2}, \cdots, b_{n}\right)$ from our $\left(v_{1}, v_{2}, \cdots, v_{n}\right)$ again by (33). Then we have, besides (34), (15), (36), which we have already observed, also

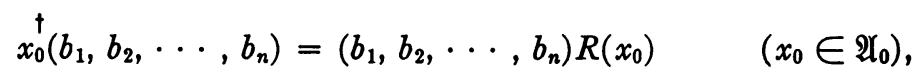

since $R=\left(Q_{1}^{\prime}\right)^{-1} R_{1} Q_{1}^{\prime}$. We obtain then

$$
3 x_{0}=Z\left(x_{0}^{\dagger}\right) \mathbb{3} \quad\left(x_{0} \in \mathfrak{A}_{0}\right)
$$

with $B$ in (13), as our analogue to (14). With $3^{*}$ in (35) we have, from (45), also

$$
\stackrel{\dagger}{x_{0} \mathfrak{S}^{*}}=\mathfrak{S}^{*} Z\left(x_{0}\right) \quad\left(x_{0} \in \mathfrak{A}_{0}\right)
$$

Consider, after all these preliminary constructions, again a right regular representation of $\mathfrak{A}$ in a decomposed form (16). It needs not, of course, be defined by our auxiliary basis $\left(u_{\imath}\right)((29))$, but we assume that it is taken such that there exists for each $\kappa$ a system of elements $\left\{e_{k j}^{(\kappa)}(i, j=1,2, \cdots, h(\kappa)\}\right.$ in $\mathfrak{A}_{0}$ satisfying (20), where $F^{(\kappa)}$ denotes the last irreducible constituent of the directly indecomposable component $U^{(\kappa)}$ in our regular representation. We take it further such that the representation matrices $F^{(\kappa)}\left(x_{0}^{\left(\dagger^{-1}\right)}\right)$ and $G^{(\kappa)}\left(x_{0}\right)$ with $x_{0} \in \mathscr{P}_{0}$, of respective degrees $f(\kappa)$ and $g(\kappa)$, coincide with each other in their places $(i, j)$ with $i, j \leqq h(\kappa)$. We also assume the absolute irreducibility of $F^{(\kappa)}$.

With such $U^{(\kappa)}$ and our above $\left(b_{\imath}\right)$, we again consider (18). We have

$$
x \mathfrak{U}^{(\kappa)}=\mathfrak{U}^{(\kappa)} U^{(\kappa)}(x), \quad \mathfrak{U}^{(\kappa)} x_{0}=U^{(\kappa)}\left(x_{0}^{\dagger}\right) \mathfrak{U}^{(\kappa)} \quad\left(x_{0} \in \mathfrak{A}_{0}\right) .
$$

The first row of $\mathfrak{u}^{(\kappa)}$ spans a left-ideal defining $U^{(\kappa)}$, and its last $f(\kappa)$ elements do not belong to the radical $\mathfrak{R}$. The same is the case with each of the first $g(\kappa)$ rows of $\mathfrak{u}^{(\kappa)}$, and for $i \leqq h(\kappa)$ the $i$ th row is congruent modulo $\mathfrak{N}$ to the first row multiplied on the right by $e_{1 \mathrm{~s}}^{(\kappa)}$. Further, each of the last $f(\kappa)$ columns of $\mathfrak{U}^{(\kappa)}$ is congruent modulo $\mathfrak{N}$ to the first of them, multiplied on the left by $e_{i 1}^{(\kappa)}$ with respective $i$; observe that $x$ is arbitrary in the first half of (49). In particular, the $(1, u(\kappa)-f(\kappa)+1)$-element of $\mathfrak{U}^{(\kappa)}$ lies again in $e_{11}^{(\kappa)} \mathfrak{A} e_{11}^{(\kappa)} \bmod \mathfrak{N}$, and is thus congruent to an element $\xi^{(\kappa)} e_{11}^{(\kappa)}\left(\xi^{(\kappa)} \in \Omega\right) \bmod \mathfrak{N}$. Here $\xi^{(\kappa)} \neq 0$ since the element is not contained in $\mathfrak{N}$. Further, for $i \leqq h(\kappa), j \leqq f(\kappa)$ we see that the $(i, u(\kappa)-f(\kappa)+j)$-element is congruent to $\xi^{(\kappa)} e_{f i}^{(\kappa)} \bmod$ $\mathfrak{N}$. Hence 


$$
\begin{aligned}
\phi_{s t}^{(\alpha)}\left(\sum_{\imath} \mu_{i j}^{(k)}\left(b_{\imath}\right) a_{\imath}\right)=\sum_{\imath} \phi_{s t}^{(k)}\left(a_{\imath}\right) \mu_{i j}^{(k)}\left(b_{\imath}\right)=\xi^{(k)} \delta_{s j} \delta_{t i} & \left(\xi^{(k)}(\in \Omega) \neq 0\right)
\end{aligned}
$$

for $i=1,2, \cdots, h(\kappa)$ (and $s, t, j=1,2, \cdots, f(\kappa)$ ). On the other hand, since $b_{\imath} \in E \mathfrak{A}$, for each $\iota, G^{(\kappa)}\left(b_{\iota}\right)$ has 0 's in its last $g(\kappa)-h(\kappa)$ rows. This shows that the left-ideals of $\mathfrak{A}$ formed by the $h(\kappa)+1$ st, $\cdots$, $g(\kappa)$ th rows of $\mathfrak{U}^{(\kappa)}$ are properly homomorphic to $\mathfrak{l}_{1}^{(\kappa)}$, the left-ideal formed by the first row. Thus $\sum_{\imath} \mu_{k}^{(\kappa)}\left(b_{\imath}\right) a_{\imath} \in \mathfrak{N}$ for $i=h(\kappa)+1, \cdots$, $g(\kappa)$. Hence (50) holds for $i=1,2, \cdots, g(\kappa)$ and $s, t, j=1,2, \cdots, f(\kappa)$ without restriction.

THEOREM 5. The orthogonality (50) holds for $i=1,2, \cdots, g(\kappa)$ and $s, t, j=1,2, \cdots, f(\kappa), F^{(k)}$ being absolutely irreducible.

Again we may consider $\mathfrak{Z}^{*}, \mathfrak{U}^{*(\kappa)}$ instead of $\mathfrak{B}, \mathfrak{U}^{(\kappa)}$. Observing (36), (48) we obtain the following theorem.

TheOREм 6. We can interchange $\left(a_{\imath}\right)$ and $\left(b_{\imath}\right)$ in the orthogonality (50) of Theorem 5 provided we consider (the normized) $V^{(k)}$ in place of $U^{(k)}$ (cf. Theorem 4); $\xi^{(k)}$ may differ from that of Theorem 5.

In fact, the argument for the case $j=h(\kappa)+1, \cdots, f(\kappa)$ is simpler than the one we had above for $i=h(\kappa)+1, \cdots, g(\kappa)$. For, we have simply $\phi_{j t}^{(\kappa)}\left(b_{\iota}\right)=0$ for those $j$.

ILLUSTRATION. Since the above construction is somewhat complicated, it is perhaps useful to illustrate it by an example. Consider the (quasi-Frobenius-) algebra $\mathfrak{A}$ consisting of matrices

$$
\left(\begin{array}{lll}
U_{1}^{(1)} & & \\
& U_{1}^{(1)} & \\
& & U_{1}^{(2)}
\end{array}\right)
$$

$$
\left(U_{1}^{(1)}=\left(\begin{array}{lll}
\beta & \gamma_{1} & \gamma_{2} \\
& \alpha_{11} & \alpha_{12} \\
& \alpha_{21} & \alpha_{22}
\end{array}\right), \quad U_{1}^{(2)}=\left(\begin{array}{lll}
\alpha_{11} & \alpha_{12} & \delta_{1} \\
\alpha_{21} & \alpha_{22} & \delta_{2} \\
& & \beta
\end{array}\right)\right)
$$

over a certain field $\Omega$. Let $e_{1}^{(1)}, e_{2}^{(1)}, c_{12}, c_{21}, e^{(2)}, d_{1}, d_{2}, d_{1}^{\prime}, d_{2}^{\prime}$ be the elements of $\mathfrak{A}$ which have respectively $\alpha_{11}, \alpha_{22}, \alpha_{12}, \alpha_{21}, \beta, \gamma_{1}, \gamma_{2}, \delta_{1}, \delta_{2}=1$ and other coefficients equal to 0 .

$$
\left(u_{\imath}\right)=\left(d_{1}, e_{1}^{(1)}, c_{21} ; d_{2}, c_{12}, e_{2}^{(1)} ; d_{1}^{\prime}, d_{2}^{\prime}, e^{(2)}\right)
$$

forms a basis of $\mathfrak{A}$, and in fact (51) is the left regular representation 
$L_{1}$ of $\mathfrak{A}$ defined by this basis (52). The right regular representation defined by the same basis is

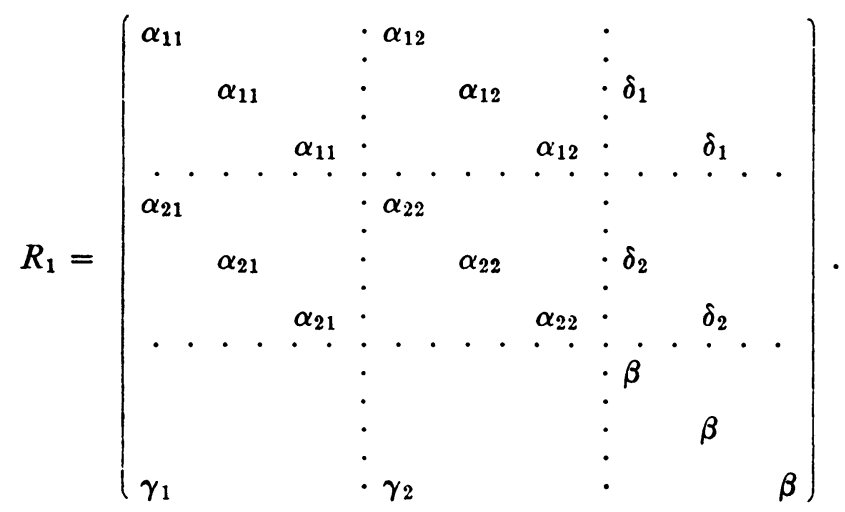

We have $f(1)=2=g(2), f(2)=1=g(1)$, and $h(1)=h(2)=1$, while $u(1)=u(2)=3$. The basis $\left(e^{(2)}, d_{1}, d_{2}\right)$ of the right-ideal $e^{(2)} \mathfrak{A}$ gives the representation $U_{1}^{(1)}$, and the bases $\left(e^{(1)}, c_{12}, d_{1}^{\prime}\right),\left(c_{21}, e_{2}^{(1)}, d_{2}^{\prime}\right)$ of $e_{1}^{(1)} \mathfrak{A}, e_{2}^{(1)} \mathfrak{A}$ give the representation $U_{1}^{(2)}$. As $\left(v_{\imath}\right)$ we can take

$$
\left(v_{\imath}\right)=\left(e^{(2)}, d_{1}, d_{2} ; 0,0,0 ; e_{1}^{(1)}, c_{12}, d_{1}^{\prime}\right) .
$$

Put $E=e_{1}^{(1)}+e^{(2)}$. Then the left and the right regular representations of $\mathfrak{X}_{0}=E \mathfrak{A} E$ defined by its basis $\left(d_{1}, e_{1}^{(1)} ; d_{1}^{\prime}, e^{(2)}\right)$ are

$$
\left(\begin{array}{llll}
\beta & \gamma & & \\
& \alpha & & \\
& & \alpha & \delta \\
& & & \beta
\end{array}\right), \quad\left(\begin{array}{llll}
\alpha & & & \\
& \alpha & \delta & \\
& & \beta & \\
\gamma & & & \beta
\end{array}\right) .
$$

Its nonsingular parastrophic matrix $P_{0}$, corresponding to the same basis, is given by, for instance,

$$
P_{0}=\left(\begin{array}{llll}
0 & 1 & 0 & 0 \\
0 & 0 & 1 & 0 \\
0 & 0 & 0 & 1 \\
1 & 0 & 0 & 0
\end{array}\right)
$$

The corresponding automorphism $\dagger$ of $\mathfrak{A}_{0}$ is given by

$$
\left(d_{1}, e_{1}^{(1)}, d_{1}^{\prime}, e^{(2)}\right)^{\dagger}=\left(d_{1}, e_{1}^{(1)}, d_{1}^{\prime}, e^{(2)}\right)\left(P_{0}^{\prime}\right)^{-1} P_{0}=\left(d_{1}^{\prime}, e^{(2)}, d_{1}, e_{1}^{(1)}\right)
$$

A conjugate basis is given by 


$$
\left(e^{(2)}, d_{1}, e_{1}^{(1)}, d_{1}^{\prime}\right) \text {. }
$$

Now, $\left(v_{\iota}\right) E=\left(e^{(2)}, d_{1}, 0 ; 0,0,0 ; e_{1}^{(1)}, 0, d_{1}^{\prime}\right)$. Augmenting (58) with 0 's as we have 0 's in $\left(v_{\imath}\right) E$, we obtain

$$
\left(z_{\imath}\right)=\left(e^{(2)}, d_{1}, 0 ; 0,0,0 ; e_{1}^{(1)}, 0, d_{1}^{\prime}\right) .
$$

Since here $\left(z_{\imath}\right)=\left(v_{\imath}\right) E$, rather accidentally, it is clear that

$$
\left(z_{\imath}\right) x_{0}\left(=\left(v_{\imath}\right) x_{0} E=L_{1}\left(x_{0}\right)\left(v_{\imath}\right) E\right)=L_{1}\left(x_{0}\right)\left(z_{\imath}\right) \text {. }
$$

Further

$$
\begin{aligned}
x_{0}\left(e^{(2)}, d_{1}, 0,0,0,0, e_{1}^{(1)}, 0, d_{1}^{\prime}\right) \\
=\left(e^{(2)}, d_{1}, 0,0,0,0, e_{1}^{(1)}, 0, d_{1}^{\prime}\right)
\end{aligned}\left(\begin{array}{lllll}
\beta & & & \\
& \beta & \gamma & \\
& & & \alpha & \\
& & & 0 & \\
\delta & & & & \alpha
\end{array}\right) .
$$

Hence

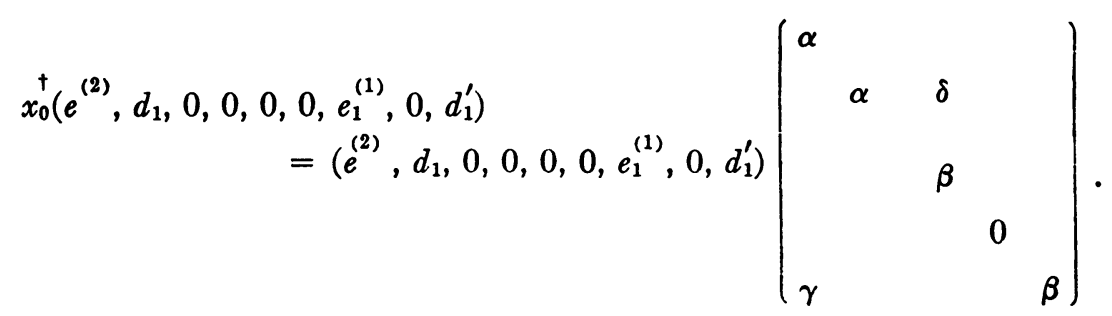

Here the 3rd (4th, 5th, 6th) and 8th rows of the matrix in the righthand side may be modified arbitrarily. Thus we have

$$
\begin{aligned}
x_{0}^{\dagger}\left(e^{(2)}, d_{1}, 0,0,0,0, e_{1}^{(1)},\right. & \left.0, d_{1}^{\prime}\right) \\
& =\left(e^{(2)}, d_{1}, 0,0,0,0, \stackrel{(1)}{e}_{1}^{(1)}, 0, d_{1}^{\prime}\right) R_{1}\left(x_{0}\right)
\end{aligned}
$$

with $R_{1}$ as in (53). The construction described above gives from $\left(z_{\imath}\right)$ $=\left(e^{(2)}, d_{1}, 0,0,0,0, e_{1}^{(1)}, 0, d_{1}^{\prime}\right)$ the same old $\left(v_{\imath}\right)=\left(e^{(2)}, d_{1}, d_{2}, 0,0,0\right.$, $\left.e_{1}^{(1)}, c_{12}, d_{1}^{\prime}\right)$.

If we take our $\left(u_{\imath}\right)((52))$ also as $\left(a_{\imath}\right)$, then $\left(b_{\imath}\right)=\left(v_{\imath}\right)$ too. We may also take $U^{(\kappa)}=U_{1}^{(\kappa)}$, though such identifications decrease the value of orthogonality. The verification of the orthogonality relations is left to readers. 
If we take, instead of the above $P_{0}$ (in (56)), the parastrophic matrix

$$
\left(\begin{array}{llll}
0 & 1 & 0 & 0 \\
0 & 1 & 1 & 0 \\
0 & 0 & 0 & 1 \\
1 & 0 & 0 & 1
\end{array}\right),
$$

then the corresponding automorphism $\dagger$ of $\mathfrak{A}_{0}$ is given by

$$
\left(d_{1}, e_{1}^{(1)}, d_{1}^{\prime}, e^{(2)}\right)^{\dagger}=\left(d_{1}^{\prime}, d_{1}-d_{1}^{\prime}+e^{(2)}, d_{1}, d_{1}^{\prime}-d_{1}+e_{1}^{(1)}\right) .
$$

The corresponding conjugate basis is

$$
\left(e^{(2)}-d_{1}^{\prime}, d_{1}, e_{1}^{(1)}-d_{1}, d_{1}^{\prime}\right) .
$$

Thus the new vector $\left(z_{\iota}\right)$ is

$$
\left(z_{\iota}\right)=\left(e^{(2)}-d_{1}^{\prime}, d_{1}, 0,0,0,0, e_{1}^{(1)}-d_{1}, 0, d_{1}^{\prime}\right) .
$$

For this new $\left(z_{\imath}\right)$ we verify also

$$
\begin{array}{r}
\left(z_{\imath}\right) x_{0}=L_{1}\left(x_{0}\right)\left(z_{\imath}\right), \quad x_{0}\left(z_{1}, z_{2}, \cdots, z_{9}\right)=\left(z_{1}, z_{2}, \cdots,\right. \\
\\
\left(x_{9}\right) R_{1}\left(x_{0}\right) \\
\left(\mathfrak{U}_{0}\right) .
\end{array}
$$

REMARK. Our orthogonality relations, for the quasi-Frobeniusalgebra $\mathfrak{A}$, are closely related to those of the Frobenius-subalgebra $\mathfrak{A}_{0}$. But they assert more than the latter do. Observe in particular that $\left(b_{\imath}\right)$ is essentially more than a basis of $\mathfrak{U}_{0}$.

REMARK. Some of our constructions and assertions can be generalized to the case where $\mathfrak{A}$ is not a quasi-Frobenius-algebra, but where the left and the right regular representations of $\mathfrak{A}$ contain certain common direct components.

\section{REFERENCES}

1. R. Brauer, On hypercomplex arithmetic and a theorem of Speiser, A. Speiser Festschrift, Zürich, 1945.

2. T. Nakayama, On Frobeniusean algebras, I, Ann. of Math. vol. 40 (1939); II, ibid. vol. 42 (1941); III, Jap. J. Math. 18 (1942).

3. T. Nakayama and C. Nesbitt, Note on symmetric algebras, Ann. of Math. vol. 39 (1938).

4. C. Nesbitt and R. Thrall, Some ring theorems with applications to modular representations, Ann. of Math. vol. 47 (1946).

NAGOYA UNIVERSITY AND

UNIVERSITY OF ILLINOIS 